Grupo de Investigaciones Jurídicas. Facultad de Ciencias Jurídicas.

Universidad Católica de Temuco.

Aabogado. Máster en Derecho Privado y Candidato a Doctor en Derecho, Universidad Carlos III de Madrid, España.

Recibido el 1 de abril de 2013, aceptado el 21 de octubre de 2013

Correspondencia a: Darío Parra Sepúlveda Grupo de Investigación Facultad de Ciencias Jurídicas Universidad Católica de Temuco.

Manuel Montt 056 Temuco-Chile. Teléfono: + 56 (45) 220 5318

E-mail:dparra@uct.cl

\section{La obligación de informar al paciente. Cuestiones sobre el derecho a ser informado}

\author{
DARÍO PARRA S. ${ }^{\mathrm{a}}$
}

This article aims to analyze, from a legal perspective, the boundaries of the obligation imposed on health care providers to inform the patient. This requirement originated and was developed as an ethical issue. However, with the newly approved law regulating the rights and duties of patients, the obligation to inform can be viewed from prisms and principles that differ from those governing medical ethics. With this purpose, we will focus on the comparative experience, which will allow us to evaluate the responsibility of health care providers when this duty is breached. We will try to answer the following questions: Which medical information must be informed to the patient? When should the doctor inform the patient? In which form should this information be provided?

(Rev Med Chile 2013; 141: 1578-1583)

Key words: Consent forms; Delivery of healthcare; Liability, legal; Malpractice.

4 n la actualidad no hay duda que las relaciones entre médico y paciente están regidas por el principio que señala que no se puede realizar una intervención quirúrgica o aplicar un tratamiento riesgoso o doloroso sin el consentimiento ilustrado y libre del interesado, salvo que la autorización no pueda ser obtenida razonablemente, o que la operación se imponga como una necesidad médica ${ }^{1}$.

En definitiva, los deberes de información están orientados precisamente a satisfacer el principio de autodeterminación del paciente. Rigen por igual si la relación del paciente con el médico se plantea en el terreno contractual, donde se trata de un deber precontractual de información del experto con el lego, o si la relación no está regida por contrato, en cuyo caso se trata de un deber general de cuidado con el paciente ${ }^{2}$.

De esta manera y como aproximación general a los deberes que tendría el facultativo relativos a la entrega de información, podemos precisar que éstos vienen a conformar los ejes centrales de una moderna forma de entender el ejercicio de la actividad médica, cambio de paradigma que para muchos vendría a compensar el tradicional y evidente desequilibrio que se producía entre el conocimiento del galeno y la ignorancia de su paciente.

\section{El deber de informar al paciente}

Tal como se ha expuesto, la relación médicopaciente es por antonomasia una relación asimétrica: el paciente acude al profesional de la medicina ante una necesidad $y$, en muchas ocasiones, motivado por desconocimiento de lo que le sucede y de cómo solucionarlo. La pregunta que surge en esta etapa apunta a determinar cómo se materializa en la práctica la obligación del facultativo de informar a su paciente. Al respecto y como primera aproximación a esta problemática, podemos destacar que el galeno deberá informar al paciente de su patología, los procedimientos a seguir, las posibilidades de tratamiento, su eventual cura y, en general, de todas aquellas prácticas que 
la correcta praxis médica establezca para el caso concreto. Es así como en esta etapa surgen como determinantes cuatro puntos básicos en la relación médico-paciente: la veracidad, la intimidad, la confidencialidad y la fidelidad.

En este sentido, cabe destacar la gran importancia que actualmente tiene la entrega de información por parte del facultativo al paciente, ya que resulta indudable que el desconocimiento de las circunstancias que rodean su enfermedad, de las posibilidades de éxito del tratamiento o intervención a la que va a someterse, así como de los riesgos que estos entrañan, coartan la posibilidad de que el paciente pueda autodeterminarse y elegir libremente si desea o no una intromisión en su propio cuerpo ${ }^{3}$.

Teniendo claro que el respeto de la autonomía de las personas se mantiene hasta nuestros días como uno de los principios más robustos de la tradición liberal en el mundo occidental, no resulta complejo observar que la protección de la autonomía personal se hace más intensa en la medida que las decisiones afectan directamente a la integridad de los propios proyectos del sujeto 4 . Por esta razón, no resulta extraño que insistentemente se haya considerado a la autodeterminación de las personas como el principio mejor conocido de la ética médica; "después de todo, la principal doctrina de esa rama de la ética es el derecho de las personas a determinar qué es lo que se hace con su propio cuerpo"s.

En este orden de ideas podemos subrayar que las exigencias de información y consentimiento tratan de fomentar la idea de autonomía individual de la persona y de estimular la toma de decisiones propias de modo racional, ayudando al paciente en la toma de decisiones, entendiendo al proceso de información no como una concesión graciable del médico, sino como un verdadero derecho del paciente ${ }^{6}$.

A este respecto, cabe recordar que la obligación de informar que actualmente pesa sobre el facultativo ha sido establecida como un deber en décadas recientes, puesto que la tradición médica ha tenido un carácter esencialmente paternalista (todo por el paciente, pero sin el paciente), que fomentaba una relación entre el médico y su paciente esencialmente vertical. Hoy, sin embargo, la relación que se trata de adoptar apunta a una forma horizontal, que respeta las circunstancias éticas y morales de cada persona que acude a los servidos sanitarios, lo cual exige poner en práctica técnicas de información ${ }^{7}$.

A continuación, reflexionaremos sobre algunas de las temáticas que se han planteado respecto de la obligación que pesa sobre el facultativo de informar a su paciente, que pueden ser englobadas en tres grandes interrogantes: a) ¿cuándo se considera que la información proporcionada ha sido suficiente?; b) ¿en qué momento debe ser proporcionada la información?; c) ¿cuál es la forma en que debe ser entregada ésta?

\section{Momento en que debe informarse al paciente}

Resulta claro que la única información eficaz es aquella que se proporciona antes de la intervención o el tratamiento de que se trate, siempre y cuando sea proporcionada con una antelación suficiente y en condiciones que permitan la adecuada reflexión por parte del paciente ${ }^{8}$. Es por ello que entre la recepción de la información y el momento en que debe dar a conocer su decisión, el paciente debe tener la oportunidad real de ponderar los beneficios, o de disponer de tiempo, por ejemplo, para recabar la opinión de familiares o de personas de su confianza'.

Por otro lado, la obligación de informar no se agota necesariamente una vez que se ha recabado el consentimiento del paciente al determinado acto médico. La manifestación de voluntad del paciente deberá estar presente durante todo el curso del tratamiento, extendiéndose incluso a las consecuencias postoperatorias y a los cuidados posteriores al acto médico que deban adoptarse ${ }^{10}$. En este orden de ideas, se ha sostenido que la información correcta no sólo concierne a la fase previa de la celebración del contrato médico, sino que también a la fase de ejecución del tratamiento acordado, por cuanto permite al paciente consentir sobre la adopción de las medidas de precaución más idóneas para la salvaguarda de su salud e integridad física ${ }^{11}$.

Otra problemática relacionada con el momento en que debe entregarse la información, dice relación con determinar si existe obligación de volver a informar a pacientes que ya han sido informados, sea porque conocen los pormenores de la intervención y sus riesgos debido a su formación o actividad profesional, o por haberse sometido con anterioridad a tratamientos o intervenciones 
equivalentes. En este punto coincidimos con la doctrina que plantea que el deber de información como obligación jurídica termina con la primera información a menos que se trate de una intervención completamente nueva, ya que guardando coherencia con el fundamento del consentimiento informado, no nos parece que deba perseguirse la responsabilidad del facultativo por no reiterar algo ya conocido y asumido por el paciente ${ }^{12}$.

En efecto, la falta de reiteración de una información que ya posee el paciente y que le permite ponderar el alcance de los riesgos y las ventajas de la intervención, no debería tener los mismos efectos que la falta de una información relevante que el paciente ignora. Ahora bien, en aquellos casos en que se presuma que el paciente dispone de una información adecuada -sea por su propia formación o por haberse sometido con anterioridad a intervenciones equivalentes- el médico tendrá la carga de comprobar que el paciente dispone de esa información razonable, de forma tal que si así no fuere la complemente en la medida en que resulte necesario. Como puede observarse, la obligación médica de información se transforma, de cierto modo, en un deber de comprobación de la información que posee el paciente a fin de determinar si ésta es completa y adecuada, siendo asumidos por el profesional los errores sobre los posibles déficits de información ${ }^{13}$.

\section{Alcance del deber de información}

En la actualidad, el deber del facultativo de entregar información a su paciente, con miras a que éste lo autorice a efectuar intromisiones en su integridad física, ha dejado de ser un mero formalismo, puesto que a través de la información suministrada por el médico, dicho paciente puede escoger con amplia libertad alguna de las opciones que la ciencia médica le ofrece, e incluso optar por no someterse a tratamiento alguno.

Si bien resulta evidente que los pacientes tienen derecho a conocer, con motivo de cualquier intervención en el ámbito de su salud, la mayor cantidad de información relevante respecto de la misma ${ }^{14}$, sobre lo que actualmente persisten vacilaciones es respecto del alcance y entidad de la información que debe ser proporcionada a los pacientes. En este sentido, y como primera aproximación a dicha problemática, podemos concluir que la obligación de informar deberá hacer referencia tanto a la finalidad y naturaleza de cada intervención, como a sus riesgos y consecuencias. En otras palabras, la exigencia de información deberá apuntar no sólo al diagnóstico, sino que también: al pronóstico, a las alternativas del tratamiento, a sus posibles complicaciones, e incluso, al carácter de adecuados o no de los medios con que se cuenta para realizar la respectiva intervención médica.

En relación a lo anterior, un importante problema que, desde la perspectiva jurídica, se ha planteado en torno al alcance que debe tener la información proporcionada al paciente, alude a la dificultad de que éste, desde su ignorancia técnico-científica, pueda comprender cabalmente las explicaciones del médico. No se trata de que el enfermo deba realizar un verdadero curso de medicina, sino que más bien se trata de conseguir, de forma racional, el objetivo que se pretende con la imposición de tal obligación al galeno, es decir, que el paciente comprenda ciertamente cuáles serán las consecuencias prácticas de su manifestación de voluntad ${ }^{3}$.

Lo anterior no quiere decir que la obligación que pesa sobre el facultativo se encuentre cumplida con la mera entrega de información al paciente. Entendiendo a la entrega de información como un presupuesto esencial para que el paciente pueda manifestar su consentimiento informado sobre una determinada intervención y teniendo presente que normalmente será el médico quien disponga de mejor acceso a la información que requiere el paciente para tomar una decisión valiosa sobre si someterse o no a un tratamiento determinado. Consideramos que este deber no debe agotarse con la simple transmisión de datos, sino que precisa, además, una cierta toma de posición de quién se encuentra legalmente obligado a proporcionar dicha información.

Así, resulta relevante indicar que el deber de información que pesa sobre el facultativo de ninguna manera ha de limitarse sólo a exponer al paciente las alternativas frente a su enfermedad, sino que además, en casos como el mencionado anteriormente, éste debe tomar una posición respecto de ellas aconsejando o desaconsejando la respectiva intervención. De esta manera, los profesionales de la salud no sólo tienen un deber de informar, entendido éste como una transmisión de datos objetivos, sino que además, y según lo requieran las circunstancias, la citada obligación conllevaría un 
deber adicional de aconsejar; esto es, de orientar a la otra parte en su decisión ${ }^{15}$.

En este sentido, debe advertirse que sólo cargar al paciente con información equivale a abandonarlo. El médico debe explicar las ventajas e inconvenientes de una y otra posibilidad diagnóstica y terapéutica, pero manifestando su opinión sobre las razones por las cuales se inclinaría hacia una u otra opción; lo contrario supone abandonar al paciente en su desconocimiento.

Dicho de otra forma, entender que el facultativo cumple cabalmente con su obligación legal sólo con la entrega de una formula ininteligible para su paciente, implicaría transformar este importante deber en una mera formalidad a cumplir, atentando claramente contra su real finalidad, la que apunta a que sea el paciente quien pueda decidir, disponiendo sobre su propio cuerpo, pero con un cabal conocimiento sobre su decisión ${ }^{16}$.

Sentado el principio anterior, resulta necesario realizar otra explicación, la cual se refiere a que debe quedar perfectamente claro que asesorar no equivale bajo ninguna circunstancia a convencer. Como lo hemos destacado, el médico, al momento de entregar la información tendría, además, el deber de asesorar a su paciente. No obstante, dicho asesoramiento sólo ha de consistir en hacerle saber a éste, de forma concreta y clara, su opinión sobre la mejor alternativa que dispone, de acuerdo a su situación y posibilidades particulares, pero bajo ninguna circunstancia el facultativo está obligado a convencer a su paciente de tomar inexorablemente un tratamiento determinado.

Por otra parte, resulta pertinente subrayar que el nivel de información exigida al galeno no siempre será el mismo, debiendo graduarse ésta en razón del tipo de información de que se trate. La idea de una graduación en la exigencia de información ha sido desarrollada principalmente en el derecho alemán cuya doctrina distingue: i) a la información relativa a la autodeterminación del paciente, es decir, aquella dirigida a obtener su consentimiento para una intervención concreta; y ii) a la denominada información terapéutica, la cual incluiría aquellas medidas a adoptar para asegurar el resultado de la intervención una vez practicada, es en este último tópico donde se encontrarían las informaciones sobre la medicación a tomar, las pruebas ulteriores que convenga realizar y medidas de prevención adicionales ${ }^{17}$.

En este contexto, cabe agregar que a efectos de determinar el nivel de rigor con que se exigirá la información en cada caso, es necesario distinguir entre medicina curativa y medicina voluntaria, puesto que los niveles de exigencias, tanto en la información relativa a la autodeterminación del paciente, como en la información terapéutica, diferirán en razón del tipo de medicina de que se hable, siendo mayores las exigencias de información cuando se trata de casos de medicina voluntaria, en comparación con los casos de medicina curativa.

A efectos de graficar brevemente la distinción entre ambos tipos de actividades médicas, cabe destacar que la primera-medicina curativa-actúa ante una determinada patología, mientras que la segunda -medicina voluntaria- es aquella en que el interesado acude al médico, no para la curación de una dolencia patológica, sino que más bien para el mejoramiento de su aspecto físico, o para lograr una transformación satisfactoria de su propio cuerpo. De esta forma, la existencia de una necesidad terapéutica, entendida como la exigencia de realizar determinadas prácticas para mantener la vida o buena salud del paciente se transforma, a nuestro entender, en el elemento fundamental para determinar en qué casos se podrá hablar de medicina curativa y en cuáles habremos de referirnos a medicina voluntaria.

\section{Forma de la información}

Un último tópico que desarrollaremos apunta a la forma en que el facultativo debe proporcionar la información a su paciente. Al respecto conviene preguntarse primeramente si ésta ha de entregarse verbalmente o por escrito. De este modo, y teniendo como principio rector el de autodeterminación del paciente, podemos concluir que, por regla general, la información entregada al paciente deberá ser enterada de forma verbal debiendo, además, ser complementada con la entrega de un folleto informativo.

Uno de los principales problemas que se ha suscitado respecto de la forma en que se pone la información a disposición de los pacientes, es la práctica de suministrarla a través de formularios o protocolos informativos genéricos. Dicha práctica ha sido ampliamente criticada, principalmente porque los formularios genéricos de información no tendrían otra función que proteger al facultativo frente a posibles reclamaciones, sin preocu- 
parse de la auténtica información que requiere el paciente, desvirtuando así la verdadera finalidad de esta importante obligación que pesa sobre el facultativo ${ }^{18}$.

A pesar del rechazo de los formularios genéricos como método único de entrega de información al paciente, resulta destacable el beneficio que implica para el paciente la entrega de un folleto informativo, siempre y cuando éste sea específico, personalizado y suplementario de la información verbal. Es por ello que nos parece conveniente optar por la utilización de folletos informativos pero sólo como un necesario complemento de la irremplazable entrega verbal de la información, debiendo destacarse en dichos volantes aquellos datos que al facultativo le interesen que el paciente tenga en cuenta al momento de tomar una decisión.

Dicho de otro modo, en la redacción de formularios hay que adoptar determinadas precauciones para que lleguen a cumplir la finalidad para la que están destinados. Será necesario que se utilicen frases cortas con abundantes puntos y aparte, palabras sencillas, evitando en la medida de lo posible los tecnicismos y sustituir éstos por su descripción con palabras comunes. Si es posible, conviene utilizar dibujos o gráficos, utilizando una estructura de texto que resulte a la vez atractiva y clarificadora. Asimismo, se deben emplear varias fuentes de letras para destacar lo que tiene una importancia esencial y una importancia secundaria; se debe favorecer el empleo de símbolos, sombreados, cajas de texto, negritas, subrayado y todos los métodos posibles que permitan la una lectura del texto lo más clara posible. Por último, debería tratar de omitirse el empleo de porcentajes numéricos así como de datos estadísticos, puesto que los datos pueden resultar muy dispares según la fuente de que procedan y según el lugar de obtención de los mismos ${ }^{19}$.

A modo de conclusión, nos parece pertinente apuntar que la reciente incursión de la ley 20.584 que regula los derechos y deberes que tienen las personas en relación con acciones vinculadas a su atención en salud, más que respuestas viene a plantear un sinnúmero de nuevas interrogantes y a recordar la discusión de algunas cuestiones todavía no resueltas, pero -a nuestro entender- la mayor revolución que trae consigo la citada normativa apunta a extraer del ámbito estrictamente bioético una serie de temáticas que hasta antes de su dictación estaban entregadas casi íntegramente a la deontología médica. De esta forma son los tribunales de justicia los encargados de ir delimitando y dando forma al conjunto de principios que han de regir la relación entre el médico y su paciente, lo cual exige que dichas problemáticas no sólo sean abordadas desde el prisma de la ciencia médica, sino que también se hacen necesarias las visiones que otros saberes pudiesen tener al respecto.

\section{Referencias}

1. Barros E. Tratado de responsabilidad extracontractual. Santiago: Editorial Jurídica, 2006; p. 682.

2. Llamas E. La llamada culpa médica: Doctrina general y especialidades problemáticas. En: Estudios jurídicos. Ministerio Fiscal 2002; p. 509-10.

3. Llamas E. La llamada culpa médica: Doctrina general y especialidades problemáticas. En: Estudios jurídicos. Ministerio Fiscal 2002; p. 510.

4. Schuck P. Rethinking Informed Consent. The Yale Law Journal 1994; 103 (4): 900-59.

5. De la Maza I. Consentimiento informado, una visión panorámica. Revista Ius et Praxis 2010; (2): 89-120.

6. Guerrero J. Las reclamaciones por la defectuosa asistencia sanitaria, Valladolid: Lex Nova, 2004; p. 47.

7. Guerrero J. Las reclamaciones por la defectuosa asistencia sanitaria, Valladolid: Lex Nova, 2004; p. 222.

8. Galán JC. Responsabilidad civil médica. Madrid: Thompson-Civitas, 2011; p. 533.

9. Katzenmeier C. Artzhaflung. Tübingen: Mohr Siebeck, 2002; p. 343.

10. García M. Aspectos básicos de la responsabilidad civil médica. Cizur Menor: Aranzadi, 2010; p. 115.

11. Galán JC. Responsabilidad civil médica. Madrid: Thompson-Civitas, 2011; p. 533.

12. Ribot J. Comentario a la STS de 22 de julio de 2003 En: Bercovitz Rodríguez-Cano, Rodrigo (Coord.) Selección de estudios jurisprudenciales de Cuadernos Civitas de Jurisprudencia Civil, Responsabilidad Extracontractual. Cizur Menor: Aranzadi, 2010; p. 135-53.

13. Ribot J. Comentario a la STS de 22 de Julio de 2003 En: Bercovitz Rodríguez-Cano, Rodrigo (Coord.) Selección de estudios jurisprudenciales de Cuadernos Civitas de Jurisprudencia Civil, Responsabilidad Extracontractual. Cizur Menor: Aranzadi, 2010; p. 151.

14. Asúa C. Responsabilidad civil médica En: Reglero Campos, Fernando (coord.). Tratado de Responsabilidad Civil, 4a Ed., Tomo II, Cizur Menor: Aranzadi, 2008; p. 753.

15. Fabre-Magnan M. De l'obligation d'information dans les contrats: essai d'une théorie. Paris: LGDJ, 1992; p. 385. 
El derecho del paciente a ser informado - D. Parra

16. Osuna E. Calidad de la información sanitaria como requisito para el consentimiento informado. Revista Latinoamericana de Derecho Médico y Medicina Legal 2000; 5 (1):59 - 65.

17. Deutsch E. Medizinrecht. Berlin: Springer, 1999; pp. 84 y ss.

18. Galán JC. Responsabilidad civil médica. Madrid: Thompson-Civitas, 2011; p. 547-48.

19. Guerrero J. Las reclamaciones por la defectuosa asistencia sanitaria, Valladolid: Lex Nova, 2004; p. 101-2. 